\title{
Cervical laminoplasty in patients with ossification of the posterior longitudinal ligaments
}

\author{
H Baba, N Furusawa, Q Chen, S Imura \\ Department of Orthopaedic Surgery, Fukui Medical School, Shimoaizuki 23, Matsuoka, Fukui 910-11, Japan
}

\begin{abstract}
Cervical laminoplasty was the operation used for myeloradiculopathy secondary to ossification of the posterior longitudinal ligaments. Some 57 patients were followed up for 5-13 years (average: 7.8 years). The spinal canal from C3 to C7 was surgically opened en bloc unilaterally with spacer bone grafting to maintain the laminae in a 'kept open' position and thus to implement posterior decompression. No serious major surgery-related complications were observed. Favourable results were obtained in 42 patients $(74 \%)$, but those with advanced preoperative neurological symptoms did not improve. Patients with spinal canals seriously compromised by anterior ossified lesions recovered poorly. We concluded that laminoplasty is recommended for cervical myeloradiculopathy due to ossified posterior longitudinal ligaments for selected patients, but surgery should be done before the patient has developed serious neurological damage.
\end{abstract}

Keywords: laminoplasty; cervical spine; myeloradiculopathy; ossification of the posterior longitudinal ligament.

\section{Introduction}

A variety of neurological symptoms is seen in patients with ossification of the posterior longitudinal ligament (OPLL) of the cervical spine. The ossified lesion frequently causes serious myeloradiculopathy which greatly handicaps the patients.

The cervical spinal cord is impinged on anteriorly and the ossified lesion continues to grow, leading to chronic compression that causes neurological damage ranging from radiculopathy alone to serious tetraparesis. Patients with minimal symptoms and signs may suffer very serious tetraplegia even after trivial cervical spine trauma. Since conservative therapy is not effective in continuous osseous entrapment, surgery is appraised anteriorly and/or posteriorly once symptoms develop. Anterior and/or posterior decompressive surgery may be appropriate depending on the modality of ossification and levels of the spinal cord compression. ${ }^{1-4}$ This paper reviews, in retrospect, posterior laminoplasty for patients with cervical OPLL myeloradiculopathy, and discusses the positive long term effects of the spinal canal enlargement.

\section{Patients and methods}

This study was originally designed to follow up 71 patients with cervical OPLL myeloradiculopathy who had had posterior laminoplasty between 1981 and 1989. Of these, six were missed in follow up, six were

This paper was presented at the 33rd Annual Scientific Meeting of the International Medical Society of Paraplegia, May 31-June 2, 1994, Kobe, Japan. excluded due to lumbosacral diseases possibly obscuring the neurological assessment of cervical OPLL lesions, and two relapsed and subsequently underwent additional anterior decompression with fusion. No other neurological disease affected follow up. Of the 57 patients available to study laminoplasty, 42 were males and 15 females. Their average age at surgery was 59 (range: $39-80$ ), and the mean follow up period was 7.8 years (range: 5-13 years). At the final follow up, patients averaged 64 years of age (range: $49-85$ ).

\section{Surgical indication and radiological assessment}

Distinct evidence of progressive myeloradiculopathy with radiographic ossification is a major indication for surgery. The OPLL lesion in a lateral radiograph was classified as segmental, continuous, mixed or circumscribed (papillary), and a magnetic resonance imaging study was done to determine levels of actual spinal cord compression. Based on the preliminary observation, ${ }^{5}$ laminoplasty was indicated for OPLL lesions involving three or more vertebrae; for lesions involving two or fewer vertebrae, anterior decompression was followed by interbody fusion. At the most seriously compromised spinal level, (in most cases being responsible for symptoms of myeloradiculopathy), the spinal canal stenosis ratio was determined in a lateral radiograph and a computed tomographic scan. The stenosis ratio was determined as the antero-posterior diameter of OPLL to the diameter of the spinal canal in the sagittal plane. The stenosis ratio was expressed as mean \pm standard deviation. For statistical treatment, a $\chi^{2}$ test was used, with $p$ value less than 0.05 viewed as significant. 


\section{Open-door laminoplasty}

The patient was positioned prone on a Mayfield 3-point head rest (Codman \& Shurtoft, Randolph MA, USA) with the neck slightly flexed to widen the interlaminar space. A standard posterior midline approach was used to expose the laminae from $\mathrm{C} 3$ to $\mathrm{T} 1$ but, for mechanical reasons, the semispinalis muscles attached to the $\mathrm{C} 2$ spinous process were preserved; these muscles are important in maintaining neck extension. Two longitudinal lateral troughs were made at sites just medial to facet joints from $\mathrm{C} 3$ to $\mathrm{C} 7$, or occasionally to T1. The inner cortices of laminae on the neurologically dominant side were usually excised using a small diamond burr and a micro-Kerrison rongeur (Eagle, Tokyo, Japan). Great care was taken not to injure the nerve roots, but operating microscopy was not needed in most cases. Contralateral hinges of laminae were thinly shaved to open the laminae en bloc. In opening the laminae, slight adhesion of the inner portion of laminae to the dura mater at the most compromised level of ossified plaque was carefully dissected with a microraspatorium. If ossification developed cranially up to $\mathrm{C} 1$ and $\mathrm{C} 2$, the caudal portion of $\mathrm{C} 2$ lamina was laminotomised for decompression (Fig 1) but, because of the wider spinal canal at C1-2, no laminectomy or major decompression was needed. Most patients showed better pulsations of the dura mater after the laminar opening. The spinous processes, harvested from $\mathrm{C} 6$ to $\mathrm{T} 1$ and about $10 \mathrm{~mm}$ long, were grafted

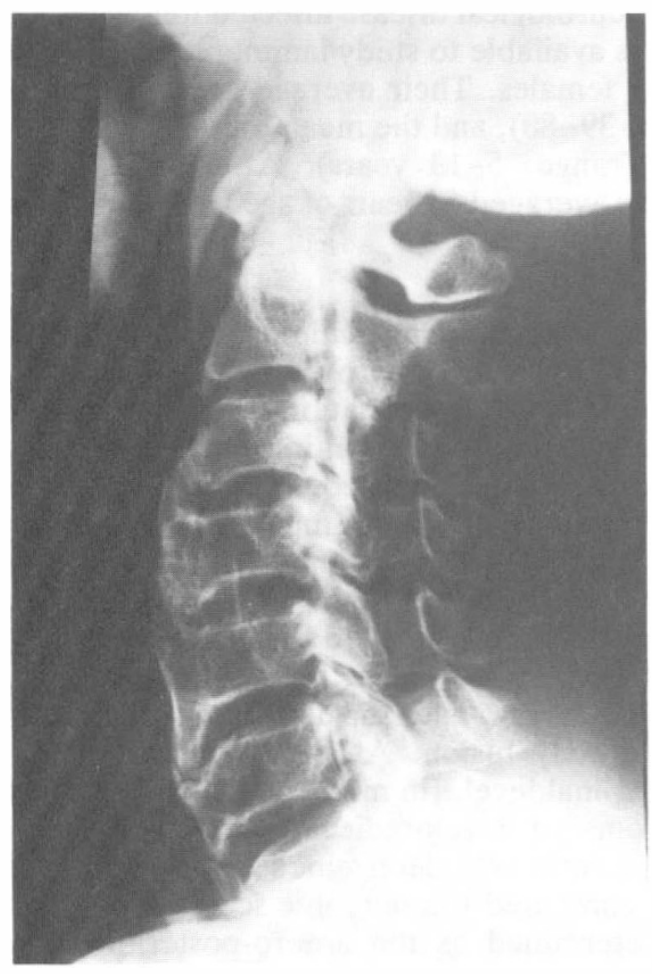

Figure 1 A 72 year old man, with OPLL from $\mathrm{C} 1$ to C5, underwent a $\mathrm{C} 3-\mathrm{C} 7$ laminoplasty with dome-like laminotomy of the inferior half of $\mathrm{C} 2$ lamina. He had made excellent neurological improvement 8 months after surgery. onto the laminae-opened space, usually at $\mathrm{C} 3$ and $\mathrm{C} 6$ levels, to secure laminae in a 'kept open' position. The harvested spinous process is trimmed as shown in Figure 2 to fix it to the cut end of the medial portion of the facet and the corresponding opened-up laminae. The ends of the bone grafts are sutured tightly to the edges of the lamina and the articular process to avoid dislodgement and loosening. This method of grafting prevents the collapse of laminae onto the spinal cord but, in most cases, due to covering by the extension muscles of the neck, a small amount of the opening is lost, as is described later. If the spinous process of the cervical spine is too small to use, corresponding material can be obtained from the upper thoracic levels. Adequate opening up of the laminae enables the dural sac to pulsate freely. In radiographs taken during surgery, the spinal canal size increased from 5 to $11 \mathrm{~mm}$ in the sagittal diameter following spacer bone grafting and, in most cases, autogenous grafts became united 6-8 months after surgery (Fig 3 ). Surgery took $1.5-3$ hours (average: 2.2 hours), blood loss was 40-370 grams (mean: 117 grams), and blood transfusion was not required for the patients. After bed rest for 3-4 days following surgery, patients were allowed to walk and were encouraged to start rehabilitation with a

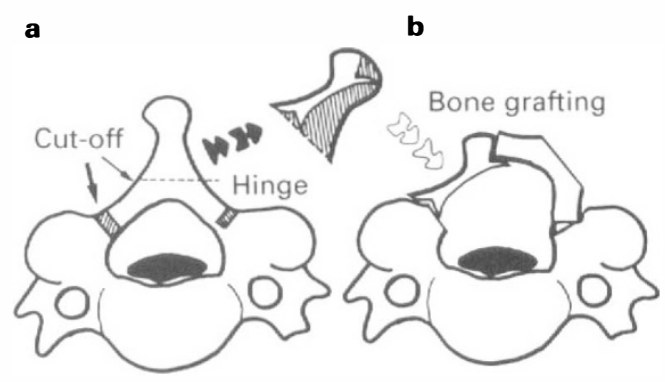

Figure 2 The technique of spacer bone grafting from the spinous process. (a) The base of the spinous process is cut transversely and then trimmed on one side each. The hatched area of the graft is resected. (b) The bone graft is inserted and fixed to the opened laminae space, and then tightly sutured.

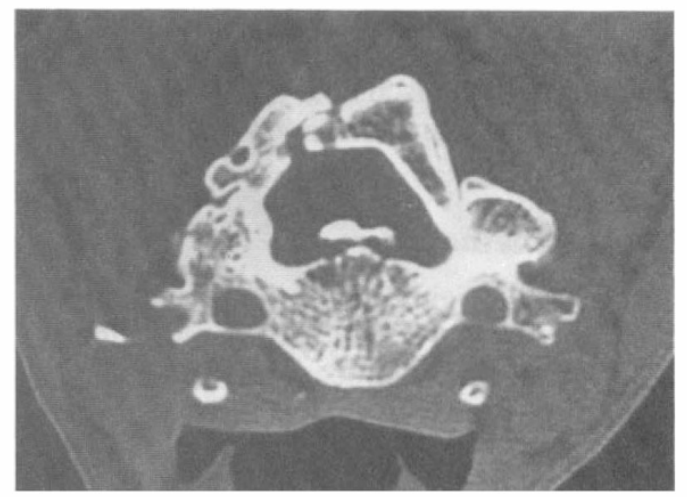

Figure 3 A computed tomography scan showing good spinal canal expansion after surgery. 
Philadelphia cervical collar (Philadelphia Co, Westville, NJ, USA). Minor complications in four patients included transient weakness in motor power in the upper extremity on the laminae opened side in two, cerebrospinal fluid leakage in one, and superficial wound infection in one. No graft dislodgement had occurred in flexion-extension radiographs taken 2-3 months following surgery, the neck collar was discontinued, and patients were allowed to engage in any sport after the bone graft had united solidly.

\section{Neurological assessment and radiographic follow up} The Japanese Orthopaedic Association (JOA) scoring system for assessing cervical myelopathy ${ }^{6,7}$ (Table I) was used in the neurological examination before and after the surgery. The postoperative improvement rate was determined as a postoperative minus preoperative score, divided by 17 minus preoperative score, multiplied by $100(\%)$. Results were excellent with an improvement rate $\geqslant 75 \%$; good $50-74 \%$; fair $25-49 \%$ and poor $\leqslant 24 \%$. At follow up, all patients showed radiographic union of spacer bone grafts and ossifica-

Table I The Japanese Orthopaedic Association (JOA) scoring system for assessment of cervical myelopathy. ${ }^{6,7}$ A completely normal condition is a total of the best scores: $(\mathrm{I}+\mathrm{II}+\mathrm{III}+\mathrm{IV})$, or 17 points

\begin{tabular}{lr}
\hline Categories & Scor \\
\hline 1 Motor function of the upper extremity & 0 \\
Impossible to eat with either chopsticks or spoon & 1 \\
Possible to eat with spoon, but not with chopsticks & 1 \\
Possible to eat with chopsticks, but inadequate & 3 \\
Possible to eat with chopsticks, but awkward & 4 \\
Normal & \\
2 Motor function of the lower extremity & 1 \\
Impossible to walk & 2 \\
Need cane or aid on flat ground & 1 \\
Need aid only on stairs & 3 \\
Possible to walk without any aid, but slow manner & 3 \\
Normal & 4
\end{tabular}

3 Sensory function

(a) Upper extremity

Apparent sensory disturbance

Minimal sensory disturbance

Normal

(b) Lower extremity

Apparent sensory disturbance

Minimal sensory disturbance

Normal

(c) Trunk

Apparent sensory disturbance

Minimal sensory disturbance

Normal

core

4 Bladder function

Urinary retention or incontinence

Severe dysuria (sense of retention, straining)

Slight dysuria (pollakiuria, retardation)

Normal tion growth was measured in the radiographs, sagittally and longitudinally.

\section{Results}

The neurological study showed that 14 patients had attained excellent, 28, good, seven, fair, and eight, poor results. Favourable neurological improvement (i.e. excellent to good) was obtained for $74 \%(42 / 57)$ of patients, averaging a $57.3 \%$ improvement rate at follow up. The preoperative neurological status significantly affected the postoperative improvement (Fig 4). Patients with advanced presurgical neurological damages could not attain better scores at follow up. Patients with severe myeloradiculopathy and a presurgical JOA score of 5 points or less $(n=12)$ improved $46.5 \%$ on the average, and those with moderate symptoms having a score of 6 to 12 points $(n=36)$ improved $58.4 \%$ on the average. Those with moderate symptoms thus improved significantly $\left(\chi^{2}\right.$ test: $\left.p<0.05\right)$. Patients with mild presurgical symptoms $(n=9)$, and a JOA score of 13 points or more had an average improvement of $53.4 \%$.

The radiological study revealed no distinct correlation between the presurgical neurological status and the stenosis ratio. Patients with mild stenosis (ratio: $<30 \%$ ) had an average presurgical score of $9.1 \pm 2.3$ points, with moderate canal compromise (ratio: $30-50 \%) 8.7 \pm 2.6$ points and with severe stenosis (ratio: $>50 \%$ ) $8.3 \pm 1.9$ points. The average improvement rate at follow up in these three groups was $60.5 \%$, $53.2 \%$, and $46.7 \%$, respectively. Patients with a $>50 \%$ spinal canal compromise showed no good recovery $\left(\chi^{2}\right.$ test: $p<0.05)$. Thirty-six patients $(63 \%$ of all) showed OPLL growth in radiographs at the level most compromised; in the mild stenosis group, OPLL grew $0.6-1.2 \mathrm{~mm}$ (mean: $0.7 \mathrm{~mm}$ ) sagittally, in the moderate stenosis group, it grew $0.5-1.3 \mathrm{~mm}$ (mean: $0.8 \mathrm{~mm}$ ), and in the severe stenosis group, it grew 0.8-2.2 (mean: $1.4 \mathrm{~mm}$ ). OPLL growth was intense in patients with severe stenosis, but not significantly so. Expanded laminae maintained the 'kept open' position satisfactorily in lateral radiographs at the follow up studies.

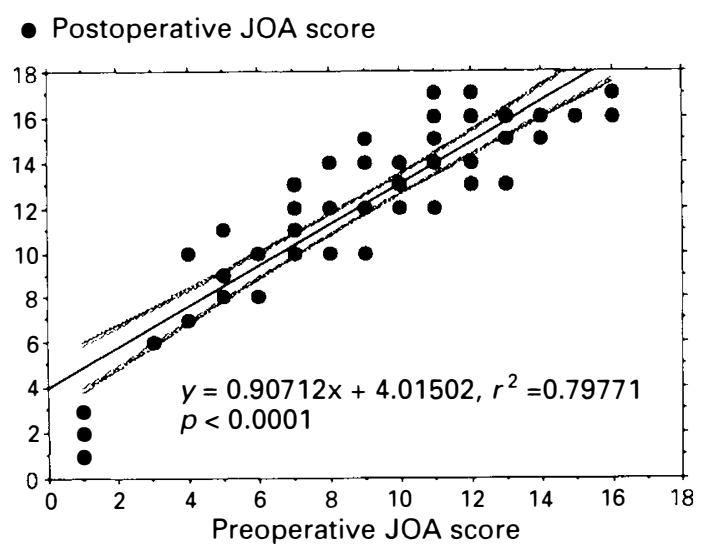

Figure 4 Correlation between pre- and postsurgical JOA scores. The score at follow up is significantly affected by presurgical neurological status. 


\section{Discussion}

In patients with cervical OPLL lesions, neurological damage progresses steadily when symptoms occur. Ventral lesions impinge persistently on the spinal cord, and compression is often multisegmental or occurs at other sites in the cervical spine.$^{8-10}$ Some reports ${ }^{11,12}$ describe OPLL as part of the established disease diffuse idiopathic skeletal hyperostosis, but OPLL is manifested radiographically quite differently from diffuse idiopathic skeletal hyperostosis: patients with OPLL frequently show no ossification or calcification in spinal ligaments other than the posterior longitudinal ligament. Despite the increase in sporadic reports on Caucasians and nonorientals, ${ }^{13-16}$ OPLL appears to have some racial predominance possibly related to generalised degenerative diseases such as diabetes mellitus and arteriosclerosis. A varying degree of neurological involvement is observed: ${ }^{17}$ recognised incidence of upper arm radiculopathy, $48 \%$; motor loss in the extremities, $10-19 \%$; and bladder dysfunction, $1 \%$. Serious tetraparesis or tetraplegia may follow minor cervical trauma. ${ }^{18}$ The cervical spinal cord loses its resistance to traumatic or additionally compressive events so that the lesion must be eliminated anteriorly, or the spinal canal be enlarged either anteriorly or posteriorly. The selection of the best surgical approach remains an open question.

Osteoplastic laminoplasty is technically simple, shorter in surgical time, is safe and promises favourable postsurgery results. ${ }^{19-21}$ Our initial observation suggests that it is preferable to laminectomy with the posterior elements for the spinal cord preserved. Because of the cervical lordosis, the spinal cord is expected to migrate away from a ventral compressive lesion following laminoplasty and thus decompress posteriorly. An alternative procedure, by an anterior approach has been advocated in selected patients. ${ }^{22,23}$ Ideally all ossified lesions would be removed anteriorly but, in multisegmental ossification, direct anterior surgery is rather difficult and adds technical risk and increased surgery time. We studied anterior resection of the ossified lesion followed by interbody fusion, hoping it could lead to a satisfactory conclusion when done, at most in two-vertebra spondylectomy. ${ }^{24}$ Anterior decompression for three or more vertebrae does little for the recovery of neurological function, possibly due to increased blood loss and unintended iatrogenic compression of the spinal cord during OPLL resection. Laminoplasty is thus justified only for OPLL involving three or more vertebrae.

The presurgical condition significantly affected postsurgical improvement; patients with a poor presurgical assessment failed to gain much following surgery. Postsurgical neurological recovery correlates significantly with the severity of the disease before surgery. We previously found that patients with severe myeloradiculopathy, i.e. a JOA score of 5 or less, did not improve after surgery, while those with moderate symptoms, i.e. a score between 6 and 12, showed significantly better neurological recovery. ${ }^{18}$ Patients with mild symptoms had similarly favourable results in relation to those with moderate paresis. This suggests that surgical intervention should be done before patients show advanced neurological symptoms, that is, possible irreversible damage to the cervical spinal cord.

One major limitation to laminoplasty is if the anterior lesion continues despite a successful posterior decompression. A huge localised ossified plaque, projecting in papillaries into the spinal canal, will continue to impinge on the canal after laminoplasty. Our study showed that patients with severe spinal canal compromise by OPLL were unlikely to recover well, suggesting that laminoplasty is ineffective in patients having severe stenosis. The lesion may also grow slowly, sagittally and longitudinally, impinging on the spinal cord continuously and thus increasing the risk of recurrence. Additional anterior decompression may be needed, especially for patients with serious cord compression and little improvement following laminoplasty. ${ }^{25}$ Most patients with OPLL myeloradiculopathy improve 6 months to a year after surgery. Two patients with localised circumscribed OPLL who underwent laminoplasty suffered recurrences due to anterior OPLL growth, requiring anterior salvage surgery. If patients show poorer recovery even after this period, anterior decompression should be considered as a salvage procedure, especially for those with circumscribed OPLL and/or a severely compromised spinal canal. In this situation, spinal cord evoked potential monitoring $^{26}$ is needed to determine the levels most responsible for the symptoms. The levels truly responsible for myeloradiculopathy are clearly delineated by epidural spinal cord evoked potentials, showing an amplitude decrementation and abnormal positive waves. Because the spinal canal had been enlarged following laminoplasty, anterior decompression for limited levels of one or two vertebrae is possible as a salvage procedure even in patients having multiple vertebral involvement.

\section{Conclusions}

Cervical laminoplasty is effective in myeloradiculopathy secondary to ossified posterior longitudinal ligaments with multilevel involvement. The more advanced the patient's neurology, the poorer the postoperative prognosis. Patients with spinal canals severely compromised by OPLL showed poor later results. For patients with severe cord compression, additional anterior decompression should be considered if the patient fails to recover or if the condition recurs.

\section{References}

1 Ono K, Ota H, Tada K et al (1977) Ossified posterior longitudinal ligament. A clinicopathologic study. Spine 2: 126-138.

2 Tsuyama N (1984) Ossification of the posterior longitudinal ligament of the spine. Clin Orthop 184: 71-84.

3 Epstein NE (1992) Management of OPLL in 24 patients treated with anterior vertebrectomy and fusion versus anterior discectomy/fusion and laminectomy. J Neurosurg 76 (suppl): 375A.

4 Epstein N (1993) The surgical management of ossification of the posterior longitudinal ligament in 51 patients. J Spinal Disord 6: $432-454$. 
5 Baba H, Imura S, Kawahara $\mathrm{N}$ et al (in press) Osteoplastic laminoplasty for cervical myeloradiculopathy secondary to ossification of the posterior longitudinal ligament. Int Orthop.

6 Tomita K, Nomura S, Umeda S, Baba H (1988) Cervical laminoplasty to enlarge the spinal canal in multilevel ossification of the posterior longitudinal ligament with myelopathy. Arch Orthop Trauma Surg 107: 148-153.

7 Japanese Orthopaedic Association (1976) Criteria on the evaluation of treatment of cervical myelopathy. J Jpn Orthop Assoc 49: Addenda No. 5.

8 Hirabayashi K, Miyasaka J, Satomi K (1981) Operative results and postoperative progression of ossification among patients with ossification of cervical posterior longitudinal ligament. Spine 6: 354-364.

9 Baba H, Tomita K, Kawahara N et al (1992) Spinal cord evoked potentials in thoracic myelopathy with multisegmental vertebral involvement. Spine 17: 1291-1295.

10 Baba H, Kawahara N, Tomita K, Imura S (1993) Spinal cord evoked potentials in cervical and thoracic myelopathy. Int Orthop 17: 82-86.

11 Resnick D, Guerra J, Robinson CA, Vint VC (1978) Association of diffuse idiopathic skeletal hyperostosis (DISH) and calcification and ossification of the posterior longitudinal ligament. J Roentgenol 131: 1049-1053.

12 Pouchot J, Watts CS, Esdaile JM, Hill RO (1987) Sudden quadriplegia complicating ossification of posterior longitudinal ligament and diffuse idiopathic skeletal hyperostosis. Arthritis Rheum 30: 1069-1072.

13 Enrique P, Charles EB, Daniel JL (1971) Ossification of the posterior longitudinal ligament associated with a herniated disc. Radiology 100: 313-314.

14 Lecky B, Britton J (1984) Cervical myelopathy due to ossification of the posterior longitudinal ligament. J Neurol Neurosurg Psychiatry 46: 1355-1361.

15 Klara M, McDonnell DE (1986) Ossification of the posterior longitudinal ligament in Caucasians: diagnosis and surgical intervention. Neurosurgery 19: 212-217.

16 McAfee PC, Regan JJ, Bohlman HH (1987) Cervical cord compression from ossification of the posterior longitudinal liga- ment in non-orientals. J Bone Joint Surg Br 69: 569-575.

17 Satomi K, Hirabayashi K. Ossification of the posterior longitudinal ligament. In: Rothman RH, Simeone FA, (eds). The spine. 3rd edn. Vol 1. WB Saunders: Philadelphia, 1992, pp 639-654.

18 Baba H, Shimada T, Imura S et al (1993) Results of surgical treatment for ossification of the posterior longitudinal ligament of the cervical spine. Abstract read at the 19th World Congress of Societe Internationale de Chirurgie Orthopedique et de Traumatologie (SICOT), April 28-September 3, Seoul, Korea.

19 Kimura I, Oh-hama M, Shingu H (1984) Cervical myelopathy treated by canal-expansive laminoplasty. J Bone Joint Surg Am 66: $914-920$.

20 Kawai S. Cervical laminoplasty. In: Bridwell KH, DeWald RH, (eds). The Textbook of Spinal Surgery. Vol 2. JB Lippincott: Philadelphia, 1991, pp 805-812.

21 Yonenobu K, Hosono N, Iwasaki M et al (1992) Laminoplasty versus subtotal corpectomy: a comparative study of results in multisegmental cervical spondylotic myelopathy. Spine 17: $1281-1284$.

22 Nagashima C (1972) Cervical myelopathy due to ossification of the posterior longitudinal ligament. J Neurosurg 37: 653-660.

23 Tominaga S (1980) The effects of intervertebral fusion in patients with myelopathy due to ossification of the posterior longitudinal ligament of the cervical spine. Int Orthop 4: 183-191.

24 Baba H, Furusawa, Tanaka Y (1994). Anterior decompression and fusion for cervical myeloradiculopathy secondary to ossification of the posterior longitudinal ligament. Int Orthop 18: 204-209.

25 Baba H, Imura S, Kawahara N, Tomita K (1994) Combined anterior and posterior surgeries for cervical myeloradiculopathy. Abstract read at the 33rd Annual Scientific Meeting of the International Medical Society of Paraplegia. May 31-June 2, 1994, Kobe, Japan.

26 Baba H, Tomita $\mathrm{K}$, Umeda $\mathrm{S}$ et al. Clinical study of spinal cord evoked potentials. In: Ducker TB, Brown RH, (eds). Neurophysiology and Standards of Spinal Cord Monitoring. SpringerVerlag: New York, 1988, pp 216-221. 